\title{
Erratum
}

\section{Sensitivity of Burkitt lymphoma Daudi cells to inactive influenza virus}

\author{
Ynon Shlomi* and Zichria Zakay-Rones \\ Department of Virology, Hebrew University-Hadassah Medical School, Jerusalem, Israel
}

J Cancer Res Clin Oncol (1989) 115: 61-66

The lines in the graphs (Figs. 1 and 2) should have been referred to by letters (a, b, c, d) in the figure legends instead of symbols.

The legend to Fig. 1 should read:

Fig. 1. Daudi cells incubated with X47 $\left(\mathrm{H}_{3} \mathrm{~N}_{2}\right)$ A Growth curve; B DNA synthesis: $a$, control uninfected Daudi cells; $c$, Daudi cells + active virus XA; $d$, Daudi cells + irradiated virus XUV; $b$, Daudi cell interferon-resistant clone + irradiated virus XUV (in A); $b$, Daudi cells + control fluid (supernatant of infected allantoic fluid after virus pelleting (in B). Daudi cells were treated with X47 viral suspensions at 100-1000 median egg-infective dose EID ${ }_{50}$ cell as described in Materials and methods. The cells were stained with erythrosin B every $24 \mathrm{~h}$, and viable and dead cells were counted (A). At different times, cells were pulse-labeled with $5 \mu \mathrm{Ci}\left[{ }^{3} \mathrm{H}\right]$ thymidine for $45 \mathrm{~min}$ and then treated as described in $\mathrm{Materials}$ and methods (B). Each point on the curve is the mean of three experiments

The Legend to Fig. 2 should read:

Fig. 2. Protein synthesis in Daudi cells incubated with $\mathrm{X} 47\left(\mathrm{H}_{3} \mathrm{~N}_{2}\right)$. $a$, Control uninfected Daudi cells; $b$, Daudi cells + active virus XA; $c$, Daudi cells + irradiated virus XUV. Daudi cells cultivated in medium without leucine were labeled with $10 \mu \mathrm{Ci}\left[{ }^{3} \mathrm{H}\right] l$ leucine and incubated with X47. At different times cells were treated as described in Material and methods 